\title{
Human Factors in Space Flight
}

Frances Mount

National Space Biomedical Research Institute

Houston, Texas 77058

\section{UNIQUE FACTORS IN SPACE FLIGHT}

After forty years of experience with human space flight (Table 1), the current emphasis is on the design of space vehicles, habitats, and missions to ensure mission success. What lessons have we learned that will affect the design of spacecraft for future space exploration, leading up to exploring Mars? This chapter addresses this issue in four sections: Anthropometry and Biomechanics; Environmental Factors; Habitability and Architecture; and Crew Personal Sustenance. This introductory section introduces factors unique to space flight.

A unique consideration for design of a habitable volume in a space vehicle is the lack of gravity during a space flight, referred to as microgravity. This affects all aspects of life, and drives special features in the habitat, equipment, tools, and procedures. The difference in gravity during a space mission requires designing for posture and motion differences. In Earth's gravity, or even with partial gravity, orientation is not a variable because the direction in which gravity acts defines up and down. In a microgravity environment the working position is arbitrary; there is no gravity cue. Orientation is defined primarily through visual cues. The orientation within a particular crew station or work area is referred to as 'local vertical,' and should be consistent within a module to increase crew productivity. Equipment was intentionally arranged in various orientations in one module on Skylab to assess the efficiency in use of space versus the effects of inconsistent layout. The effects of that arrangement were confusion on entering 
the module, time spent in re-orientation, and conflicts in crew space requirements when multiple crew members were in the module.

Design of a space vehicle is constrained by the three major mission drivers: mass, volume and power. Each of these factors drives the cost of a mission. Mass and volume determine the size of the launch vehicle directly; they can limit consumables such as air, water, and propellant; and they impact crew size and the types of activities the crew performs. Power is a limiting factor for a space vehicle. All environmental features - e.g., atmosphere, temperature, lighting - require power to maintain them. Power can be generated from batteries, from fuel cells, or from solar panels. Each of these sources requires lifting mass and volume from Earth, driving mission cost. All engineering decisions directly impact the design for habitation design and usage. For instance, if fuel cells are used they produce water, which is used for drinking and food preparation. If a different power source is used water has to be carried and stored on the vehicle which then directly impacts the food system choice as well as the launch weight of the vehicle.

The size of the crew directly affects mass and volume requirements. The larger the crew, the larger the vehicle. Crew time is expensive and is recognized as another mission driver. Designing equipment and procedures to maximize returns from crew time must be considered in the earliest stages of mission planning to ensure mission success. Detailed studies of how crew time was actually used during Skylab (Bond, 1977) showed that approximately 1/3 of the crew time was spent in sleep, close to $1 / 3$ in other forms of self-sustenance such as hygiene, exercise, eating, recreation, and more than 1/3 was actually devoted to operating the spacecraft and scientific experiments.

Habitability and human factors requirements are strongly driven by mission duration and the opportunity for resupply or emergency return to Earth. The Space Transportation System (STS) was designed for missions on the order of two weeks - analogous to a camping trip. With Mir and the International Space Station (ISS), mission durations of six months became standard, requiring far more concern for 
habitability and for crew efficiency, training, and sustenan ce. As NASA begins to plan for a mission to the Mars surface, with travel times on the order of six months each way and a possible surface stay of 18 months, support and services to crew members must be addressed. Topics of concern include: health maintenance, training, recreation, food, clothing, vehicle maintenance, science tasks, etc.

To date, the model for space exploration has had a very small crew - from a maximum of seven or ten on a Shuttle flight, to just two people on the ISS - supported by a very large group of scientific and engineering experts on the ground, linked through the Mission Control Center (MCC). This model has been essential because such a small crew cannot be expert in all the critical subsystems on board. There are too few people to understand the subsystems in sufficient detail to operate and maintain them under nominal circumstances, let alone when malfunctions occur. But this model depends on rapid two-way communications. Video and audio transmissions allow the MCC to see and hear the crew, and to transmit questions and procedures in a short enough time to be responsive to time-critical events. Even on the lunar surface, communications lags are just on the order of seconds. With a mission to Mars, the nature of communications and the roles of the ground and flight crews will be reexamined to consider a delay of 20 minutes each way.

\section{ANTHROPOMETRY AND BIOMECHANICS}

In a microgravity environment the body changes. Immediately on reaching free-fall, the body assumes a 'neutral' posture quite different from standing or sitting postures on Earth. The neck, shoulders, elbows, hips and knees all flex somewhat, and the shoulders also abduct and rotate with large inter-subject variability. The result affects the crewmember's line of sight, height, and reach envelope. The range of postures observed on a specific flight is shown in Figure 1. Figure 2 illustrates reach envelopes based on a typical posture for a 95th percentile crew member.

After a short while in microgravity - on the order of hours - the body height changes due to spinal elongation, which is caused by the lack of gravity. Height increases about $3 \%$ during the first day or so in 
microgravity. The distribution of body fluids also changes. Greater amounts of body fluids move to the head and torso, affecting hand size, facial appearance, the voice, taste, and perhaps the sense of smell. Suits and gloves used for tasks outside the vehicle (Extra Vehicle Activity or EVA), must fit snugly, and therefore must accommodate the physical changes.

As crew members attempt to perform physical tasks they push themselves away from the workplace. Without gravity they need to be physically restrained to perform work. The most common restraint for crew members is a foot restraint. For a location where a person will be working for extended periods of time, there are platforms that can be tilted to accommodate the neutral posture, with the feet angled down, and with height adjustments.

Tasks of different durations and requiring different degrees of force or dexterity require different types of restraints. Frequently short, easy tasks can be performed with toes stuck under a handle or one hand on a handhold. Tasks such as attaching a module to the ISS using the remote manipulator system, which take many hours and high precision of hand-eye coordination, require a restraint such as shown in Figure 3 . This restraint provides support for the feet and the thighs. Another example of restraints is shown in Figure 4, illustrating use of existing hardware for a temporary restraint.

\section{ENVIRONMENTAL FACTORS}

When designing a space mission, every effort is made to recycle air and water rather than to carry replacements on a mission. In a long-term mission to a planetary surface, carrying air and water would be prohibitive. This affects the design of the habitat and equipment. For a closed loop system, any and all materials used in the vehicle must be compatible with this closed system and therefore cannot release compounds that are difficult to remove from the atmosphere. Materials used also must be compatible with cleaning materials and biocides that are safe for the environment without outgassing. Materials must not encourage flourishing colonies of bacteria and mold. Mission planning must take into account all considerations for atmospheric conditions and balance them with the constraints of the mission: mission length; mission objectives; requirement for pre-breathe for EVA; mission research requirements; and 
equipment in the vehicle.

Drinking water is the first requirement for water during a mission. In addition to this obvious need water is required for a variety of other uses. They include food preparation, personal use, hygiene and housekeeping. If plants are to be grown during the mission, that creates an additional water requirement. The amount of water for food preparation is dependent on the food system type selected. Typical water requirements for drinking, hygiene and washing for each crewmember are 2.84 to $5.16 \mathrm{~kg}$ per person per day for standard operational mode. (NASA, 1995). Water that is reclaimed and stored depends on its quality for its use. A crew depends on water that is clean and safe.

From the perspective of human factors, noise can affect performance by interfering with communications, interfering with sleep, and causing annoy ance. Constant noise above acceptable levels can result in hearing loss. In an assessment of the SpaceHab-1 mission (STS-57), Mount et al. (1994) found that while the measured noise levels did not generally exceed the permitted levels for the shuttle flight deck or middeck, noise levels were substantially above design limits for the SpaceHab. Space vehicles present a significant acoustics challenge because the modules have equipment such as fans, pumps, compressors, avionics, and other noise producing hardware or systems to serve their functional and life support needs all in a confined volume.

Lighting is essential to performing virtually every task in space. When windows are present and unshuttered, the typical $90 \mathrm{~min}$. low earth orbit of the Shuttle or Station cause problems with insufficient time for eyes to adapt to the rapid disappearance of sunlight. In the study by Mount et al. (1994) the most frequent report of lighting problems was that sunlight made electronic displays and video monitors difficult or impossible to read. Windows are necessary for some activities, such as remote manipulator operations. Earth-watching is a favorite crew activity in any spare time.

For future Lunar / Mars exploration missions, the problem of dust in these environments is recognized. 
However, our knowledge at this time is limited as to the specifics of the dust. We have some data from previous Lunar Missions and are supplementing it with derived data. Derived data from our limited, but growing, knowledge of Mars is forming a basis of our need for requirements for dust abatement. The dust will cause a serious problem for EVA suits and equipment used external to the vehicle. There is also a concern of dust in the vehicle habitation area. Dust inside the vehicle could increase crew time due to more frequent filter changes and other chores to remove dust from equipment. Basic habitability could also be affected if the dust were to accumulate inside the vehicle on display screens, cooking equipment and other places.

\section{HABITABILITY AND ARCHITECTURE}

A designer for a space vehicle must provide a safe, comfortable, functionally efficient habitat that will support mixed crews living and working together for the duration of a mission. This crew could be of various cultures and backgrounds. Mission duration could be days or years. Attention must be given to the crew morale, comfort, health, and physical size. Any architecture and design of interior components should be comfortable for the crew member of average size, but should not be uncomfortable for the extremes of any crew population. The "Habitability Architecture" design concerns are mainly with respect to the fixed architectural elements of the crew interior interfaces such as (a) the geometric arrangements

of compartments (b) passageways and traffic paths., (c) windows (d) color, (e) workstations, (f) off-duty, (g) stowage, (h) lighting. (NASA, 1983)

All habitable volumes should include windows that are adequate for terrestrial and celestial references. Windows are necessary for observation of scientific phenomena, monitoring of EVA, observation of the vehicle exterior, photography, and general viewing. Sufficient window locations should always be provided to view earth, both for Earth observation experiments and for crew recreation and well-being. Historically, the main recreation for orbiting crew has been observing Earth. 
Any location in the space vehicle where a dedicated task or activity is performed exclusive of the recreation, personal maintenance, and sleep areas, is defined as a workstation. Tasks and activities include: Vehicle stabilization and control, Systems management, Experiments, Science, and Maintenance (equipment repair), EVA monitoring, Earth observation, and food preparation. With any workstation, analysis should be done to determine the tasks, operator activities, tools and equipment necessary for each workstation. To make efficient use of space, multi-use workstation can be considered. All necessary equipment, tools, restraints, lights, and power outlets should be provided at each workstation. Adequate space should be provided for the crew to perform the assigned tasks efficiently and safely. Where possible, standardization of workstations is a benefit for design of associated equipment. Where possible, commonality of displays should be provided for efficiency.

Off-duty activities should have a dedicated area with a minimum space for the entire crew. This allows for socialization. Stowage areas should be provided in a dedicated recreation area and in the personal space area for items to be used during recreation activity and off-duty time. (NASA, 1983) There has been agreement from crewmembers on U.S. Missions and also from crew during analog studies that they do not like to use the same table for dining as well as for maintenance tasks and / or for biology tasks. (Mount, 2002)

With any space vehicle design for a long-term mission, an area for recreation should be designated to provide for social interaction, Earth-viewing, games, video tape viewing, music, and active and passive participatory activities. A quiet area should be provided for a crewmember to read, listen to music and write.

Stowage must be compatible with the type of mission planned. A long-term mission with no resupply would have a different stowage plan than a shorter mission with regular resupply. Stowage space must be provided based on planned need. For efficient use, the stowage space should be near the areas where the stowed items will be used. A method should be provided for locating stowed equipment and supplies and 
monitoring their use level. This is extremely important for a mission like the International Space Station where crews are periodically changed out, but large quantities of the stowed equipment and supplies stay. (NASA, 1983) Means should also be supplied to adequately stow trash and expired items. For long duration missions in which routine resupply is required (e.g., ISS) habitat engineers should design to manage the stowage of a large influx of supplies - which can encroach significantly on crew habitable volume. A system for ready integration of the new supplies into the existing stowage system ensures limited shelf-life consumables and critical equipment are easily accessible.

The basic lighting requirements are no different for space vehicles than for habitat design on Earth. Spacecraft designers face a few human factors' challenges not usually encountered in earthbound environments. In general, design of any space vehicle must take into account the constraints of power and weight. This has an impact on the number of lights and their specifications. The use of fixed luminaires for general illumination within the relatively small habitable volume of a spacecraft implies that an astronaut may frequently find one or more of these light sources in her/his field of view as she/he floats in microgravity, thus creating potential direct glare sources.

Unintentional contact with surfaces and edges has a much greater likelihood of occurrence in space. Since a crew member can be moving through an area in a unique way, 'floating', care must be taken that surfaces, edges, clamps, fasteners, etc. have no sharp edges. This includes edges and corners that a crew member may be exposed to during a maintenance activity. While a crew member is translating by floating, anything can be grabbed onto and used as a handhold, or kicked, or bumped into. Additionally, since random grabbing, bumping or kicking may occur, touch temperature is important to keep with safe limits. Switches, knobs, dials, etc. should be protected against activation by inadvertent grabbing, hitting, or kicking during a pass by

\section{CREW PERSONAL SUSTENANCE}

The spacecraft must be designed to provide for all aspects of life. For long duration missions, private 
compartments are used for sleep and certain personal activities such as recreational reading or communicating with family and friends. An individual sleep compartment should be provided for each crewmember. Since the sleep compartment is the single location in which the crew member spends the most time, it has been found to be most effective to heavily shield the compartment against radiation. The private sleeping accommodations should have a privacy curtain, partitions and stowage lockers. Each sleep area should be located as far as possible from noise, activity and public area.

Since there is no up or down in weightlessness, the position of the body does not matter during sleep (Figure 5). Some astronauts have been bothered by an effect known as 'head nod'. If the head is not secure when fully relaxed during sleep the head develops a nodding motion. Astronauts can secure the sleep restraint (sleeping bag) to limit this nod. Skylab sleep restraints were similar to sleeping bags with neck holes and arm slits. Straps were on the front and back so the crewmember could be tightened for a steady, snug position. The Space Shuttle missions sometimes split the crew into two shifts to enable around the clock science. Figure 6 shows the compartments provided for the off-duty crew's sleep area.

Since the first food was consumed in orbit in 1962, improvements and developments have been made and are continuing to be made in the food systems for manned space flight. The food system for the Mercury flights was limited in scope and purpose. Food was used in most cases to obtain general information on the effects of null gravity on food ingestion and digestion and to determine types of food and packaging for longer duration space flights. Results from Apollo proved that food could be consumed from an open container using normal utensils in micro-gravity.

At present, research is ongoing to look into advanced technology for future food systems for Lunar and / or Mars long-term missions. This includes the growing of crops onboard a space vehicle. The type of food as classified by the method of preservation also influences the weight of the food system, the palatability and the preparation. Table 2 shows the support elements or activities associated with each classification of preservation. Most of the natural form foods such as cookies, dried fruits, etc. only 
require opening the package compared to the dehydrated foods which require six support elements. The paramount importance of the food system in a long-duration manned exploration mission should not be under-estimated. During long-duration space missions, several physiologic effects may occur. They include weight loss, fluid shits, dehydration, constipation, electrolyte imbalance, calcium loss, potassium loss, decreased red blood cell mass, and space motion sickness. The menu will provide the crew with changes in the nutrient levels that may be required due to the longer-duration mission. The acceptability of the food system is of much greater importance due to the longer-mission durations and the partial energy intake that is often observed in space flight. The decreased energy intake might significantly compromise the survival of the crew.

Managing personal waste and cleaning the skin and hair are problematic because of the lack of gravity and the cost of lifting water to orbit. Except for Skylab, dedicated volumes for various activities have been very limited. Early bodily waste management systems can be succinctly described as 'baggies'. Since Skylab, there have been a variety of suction-based toilets for collecting fecal matter and urine.

Exercise regimens prescribed for space missions have required gradually longer and more frequent periods of exercise - particularly as the length of mission has increased. Results of exercise on past missions have shown a similar pattern of reduced physiological deconditioning in response to more strenuous exercise programs.

\section{CONCLUSION}

After forty years of human space flight we have gathered great quantities of information dealing with the crew and their interfaces. With new missions in front of us, going beyond low earth orbit, we must learn more about the challenges of long term missions. We must gather much more data from ISS missions. Additionally, we must take advantage of analogs that are well defined and consistent with the perceived challenges of long-term missions, and glean what we can to augment our knowledge base. 


\section{REFERENCES:}

Bond, R.L. (1977) “Application of Skylab Workday Analysis to Future Programs,” JSC 12856. Johnson Space Center, Houston, TX.

Mount, F.E., (1989), “Session Summary: Maintenance”, in Proceedings of The Manned System - A Human Factors Symposium and Workshop, American Astronautical Society, Houston, TX.

Mount, F.E., Adam, S., McKay, T., Whitmore, M., Merced-Moore, D., Holden, T., Wheelwright, C., Koros, A., O’Neal, M., Toole, J., and Wolf, S. (1994) “Human Factors Assessments of the STS-57 SpaceHab 1 Mission. NASA Technical Memorandum 104802.

Mount, F.E. (2002), "Habitability: an Evaluation" in Isolation: NASA Experiments in ClosedEnvironment Living, H.W. Lane, R.L. Sauer, and D.L. Feeback, Eds., Univelt, San Diego, Pp. 87-116

National Aeronautics and Space Administration, (NASA) (1983), "Crew Interface Panel Space Station Habitability Requirements Document," JSC 19517, Johnson Space Center, Houston TX

National Aeronautics and Space Administration, (NASA) (1995). Man-Systems Integration Standards, NASA-STD- 3000.

National Aeronautics and Space Administration, (NASA), (2003), Astronaut Fact Book, NP-2003-07008JSC, Johnson Space Center, Houston TX. 